\title{
Energy-Based Model-Reduction of Nonholonomic Mechanical Systems
}

\author{
Vincent Duindam \\ Control Engineering, EEMCS, University of Twente \\ P.O. Box 217, 7500 AE Enschede, The Netherlands \\ v.duindam@icee.org
}

\author{
Stefano Stramigioli \\ Control Engineering, EEMCS, University of Twente \\ P.O. Box 217, 7500 AE Enschede, The Netherlands \\ s.stramigioli@ieee.org
}

\begin{abstract}
Research on nonholonomic mechanical systems has focused mainly on describing geometric structure, controllability, and motion planning, yet little attention has been paid to several energy aspects of these systems. This paper describes a method to model nonholonomic mechanical systems as reduced-order PortControlled Hamiltonian Systems, in which the energy structure is shown explicitly. We show how very simple equations are obtained for the example of the snakeboard, and then discuss how these equations can be used to derive an energy-based controller in an intuitive way.
\end{abstract}

\section{INTRODUCTION}

Research on locomotion systems has always been inspired by nature. In nature, animals locomote by periodically changing their internal shape such that an overall displacement is obtained. This overall displacement can usually be described by a group motion (since the motion is described by an element of the appropriate mathematical group, like $S E(2)$ for planar systems). The reason for this net motion is the presence of constraint forces from the environment; the reason that a car can accelerate is that the ground applies constraint forces on the wheels such that they do not slip. Even though these constraint forces do no mechanical work, they make it possible to convert energy generated internally in the shape to energy in the group, i.e. forward momentum.

Constraints such as these are called kinematic constraints as they forbid certain velocities and allow others. Kinematic constraints that cannot be reformulated as configuration constraints (i.e. non-integrable constraints) are called nonholonomic constraints, and these are precisely the type of constraints that are interesting for locomotion.

A well-known example of a nonholonomic system is the snakeboard [1], a locomotion device similar to the skateboard. This system is relatively simple to describe, yet possesses many features that are interesting from a modeling and control point of view: it has nonholonomic constraints, symmetries (invariances), and is underactuated. Through certain combinations of the control actions and with the help of the constraint forces, it is possible to build up momentum in directions that are not directly actuated. This behavior has been studied using different techniques, e.g. Lie-bracket analysis [2], [3], averaging [4], [5], principal bundles [6], and momentum maps [6], [4], [7]. These studies have revealed many interesting physical and geometric properties and interpretations, and provide general tools to analyze nonholonomic systems.
However, one aspect that has received relatively little attention, is the energy flow in these systems, especially the energy flow from the controller. During the analysis of the snakeboard in the before-mentioned references, usually feedbacklinearization is applied to the directly controlled states, which makes energy analysis impossible. But with applications like efficient locomotion in mind, analyzing the energy aspects of the system may be very important.

In this paper, we approach the modeling and control of nonholonomic systems from the viewpoint of Port-Controlled Hamiltonian Systems, because that way the energy properties can be expressed explicitly. We show how a mechanical system with nonholonomic constraints (like the snakeboard) can be transformed into a Port-Controlled Hamiltonian System without constraints in a reduced number of variables, and how this model can be used for energy-based control purposes. The reduced-order model for the snakeboard turns out to be remarkably simple, and appropriate controllers can be designed quite intuitively.

This paper is organized as follows. Section II discusses the necessary mathematical background. Section III describes the dynamics and constraints of the snakeboard. Section IV contains the derivation of the reduced-order equations for general nonholonomic systems, and Section V discusses how these reduced equations can be used to analyze and design a controller for the case of the snakeboard. Finally, Section VI gives the main conclusions of the paper and describes possible directions for future research.

\section{MATHEmatical. BACKgROUND}

\section{A. Manifolds and Tensors}

We denote a differentiable manifold by $Q$, its points by $q$, and its dimension by $n \in \mathbb{N}$. The tangent bundle $T Q$ of $Q$ is the union of the tangent spaces $T_{q} Q$ at all points $q \in Q$. Similarly, the cotangent bundle $T^{*} Q$ of $Q$ is the union of all cotangent spaces $T_{q}^{*} Q$. The intrinsic dual product between an element $v \in T Q$ and an element $\alpha \in T^{*} Q$ is denoted by $\langle v \mid \alpha\rangle \in \mathbb{R}$.

A $\mathcal{C}^{\infty}$ tensor field $T_{(l)}^{(k)}$ is a $\mathcal{C}^{\infty}$ mapping which assigns to each point $q \in Q$ a tensor of order $k$ contra-variant and order 
$l$ co-variant (a type $(k, l)$ tensor) such that the mapping

$$
T(q): \underbrace{T_{q} Q \times \ldots \times T_{q} Q}_{l \text { times }} \times \underbrace{T_{q}^{*} Q \times \ldots \times T_{q}^{*} Q}_{k \text { times }} \rightarrow \mathbb{R}
$$

is linear in all its arguments at all $q \in Q$. Tensor fields can locally be expressed using coordinates, e.g. $T_{v w}^{x y}$ expresses the value of $T$ acting on the basis vectors $\partial_{v}, \partial_{w} \in T Q$ and $d x, d y \in T^{*} Q$. We use the Einstein summation convention, which means that repetition of an index (once upper, once lower) implies summation over that index. Furthermore, we denote the partial derivative of a tensor $T_{v w}^{x y}$ to $q^{i}$ by $T_{v w, i}^{x y}$

A Riemannian metric tensor field (denoted by $g$ or in coordinates by $g_{i j}$ ) assigns to each point a symmetric positivedefinite two-covariant tensor. A manifold endowed with such a structure is called a Riemannian manifold. Using the metric, we denote the inner product of two tangent vectors as

$$
\langle v, w\rangle_{g}=g_{i j} v^{i} w^{j} \in \mathbb{R} \quad v, w \in T_{q} Q
$$

The inverse of the metric defines a metric $g^{-1}$ on $T_{q}^{*} Q$ as

$$
\langle\alpha, \beta\rangle_{g^{-1}}=g^{i j} \alpha_{i} \beta_{j} \in \mathbb{R} \quad \alpha, \beta \in T_{q}^{*} Q
$$

\section{B. Port-Controlled Hamiltonian Systems}

A general conservative explicit Port-Controlled Hamiltonian System (PCHS) is a dynamical system that can be represented by a set of differential equations of the following form

$$
\begin{aligned}
& \dot{x}=J(x) \frac{\partial H}{\partial x}+g(x) u \\
& y=g^{T}(x) \frac{\partial H}{\partial x}
\end{aligned}
$$

in which $x \in \mathcal{X}$ is the state of the system, $H$ is the (differentiable) energy function, $J(x)$ is a skew-symmetric matrix called the structure matrix, and $(u, y) \in \mathcal{U} \times \mathcal{U}^{*}$ is the port through which the system can interact with e.g. a controller. For systems of this form it is straightforward to show that $\dot{H}=\langle u \mid y\rangle$, i.e. the increase in internal energy is equal to the power supplied through the port $(u, y)$. Several generalizations for this kind of systems exist to include e.g. dissipation and implicit formulations, but we do not consider those general systems here. We refer the interested reader to [8].

In this paper, we consider the subclass of mechanical systems (with $H$ the mechanical energy) and we start from a so-called simple mechanical system (a system for which the total energy is the sum of kinetic and potential energy). If we take the state to be an element of the cotangent bundle $T^{*} Q$, the dynamics can be described by a PCHS of the form

$$
\begin{aligned}
\frac{d}{d t}\left[\begin{array}{l}
q \\
p
\end{array}\right] & =\left[\begin{array}{cc}
0 & I \\
-I & 0
\end{array}\right]\left[\begin{array}{l}
\frac{\partial H}{\partial q} \\
\frac{\partial H}{\partial p}
\end{array}\right]+\left[\begin{array}{l}
0 \\
B
\end{array}\right] u \\
y & =\left[\begin{array}{ll}
0 & B^{T}
\end{array}\right]\left[\begin{array}{l}
\frac{\partial H}{\partial q} \\
\frac{\partial H}{\partial p}
\end{array}\right]
\end{aligned}
$$

where $(q, p)$ are the canonical coordinates of the cotangent bundle (generalized positions and momenta), and $H$ equals

$$
H(q, p)=\frac{1}{2}\langle p, p\rangle_{g^{-1}}+V(q) .
$$

The first term of $H$ is the kinetic energy, the second term is the potential energy. Systems described in these coordinates $(q, p)$ with $J$ as shown are called symplectic systems.

\section{Nonholonomic Kinematic Constraints}

In addition to the control port $(u, y)$, we allow the environment to exert constraint forces on the system, such that velocities are constrained in certain directions. There are several ways to represent these constraints; here, we require that the velocities $\dot{q}$ of the system belong to a smooth distribution $\mathcal{D} \subset T Q$ with constant dimension $m$. This means that we only look at constraints that are linear in the velocities. If $\mathcal{D}$ is integrable, then by Frobenius's Theorem [9] we can find coordinates on $Q$ such that satisfying the constraints is equivalent to some of these coordinates being constant. In other words, the constraints on the velocities can be expressed equivalently as constraints on the configurations. Such integrable constraints are called holonomic, whereas general non-integrable constraints are called nonholonomic. In this paper, we allow for nonholonomic constraints.

From the distribution $\mathcal{D}$ and using the metric $g$, we can define a decomposition of $T Q$ and a corresponding decomposition of $T^{*} Q$ as follows. Given $\mathcal{D}_{q}$, we define $\mathcal{C}_{q}$ pointwise as

$$
\mathcal{C}_{q}:=\left\{v \in T_{q} Q \mid\langle v, h\rangle_{g}=0 \quad \forall h \in \mathcal{D}_{q}\right\}
$$

$(\mathcal{C}$ is the $g$-orthogonal complement of $\mathcal{D}$ ). We can now decompose $T_{q} Q$ as

$$
T_{q} Q=\mathcal{D}_{q} \oplus \mathcal{C}_{q}
$$

and in a dual way decompose $T_{q}^{*} Q$ as

$$
T_{q}^{*} Q=\mathcal{C}_{q}^{\perp} \oplus \mathcal{D}_{q}^{\perp}
$$

where

$$
\begin{array}{ll}
\mathcal{D}_{q}^{\perp}:=\left\{\alpha \in T_{q}^{*} Q \mid\langle\alpha \mid h\rangle=0\right. & \left.\forall h \in \mathcal{D}_{q}\right\} \\
\mathcal{C}_{q}^{\perp}:=\left\{\alpha \in T_{q}^{*} Q \mid\langle\alpha \mid v\rangle=0\right. & \left.\forall v \in \mathcal{C}_{q}\right\}
\end{array}
$$

To incorporate the constraint forces in the Hamiltonian model, we extend (3) and (4) to

$$
\begin{aligned}
\frac{d}{d t}\left[\begin{array}{l}
q \\
p
\end{array}\right] & =\left[\begin{array}{cc}
0 & I \\
-I & 0
\end{array}\right]\left[\begin{array}{l}
\frac{\partial H}{\partial q} \\
\frac{\partial H}{\partial p}
\end{array}\right]+\left[\begin{array}{l}
0 \\
B
\end{array}\right] u+\left[\begin{array}{l}
0 \\
\mathcal{F}
\end{array}\right] \\
y & =\left[\begin{array}{ll}
0 & B^{T}
\end{array}\right]\left[\begin{array}{l}
\frac{\partial H}{\partial q} \\
\frac{\partial H}{\partial p}
\end{array}\right]
\end{aligned}
$$

where $\mathcal{F} \in \mathcal{D}^{\perp}$ are the constraint forces which are assumed to do no mechanical work, i.e. the constraints are ideal [6]. Note that this definition is slightly different from the traditional one, in which the constraint forces are added as $\mathcal{F}=A^{T}(q) \lambda$ so the space $\mathcal{D}^{\perp}$ is explicitly described by the image of $A^{T}(q)$. The space $\mathcal{D}$ of allowed velocities is then dually described by the kernel of $A(q)$, i.e. the constraints are $A(q) \dot{q}=0$. 


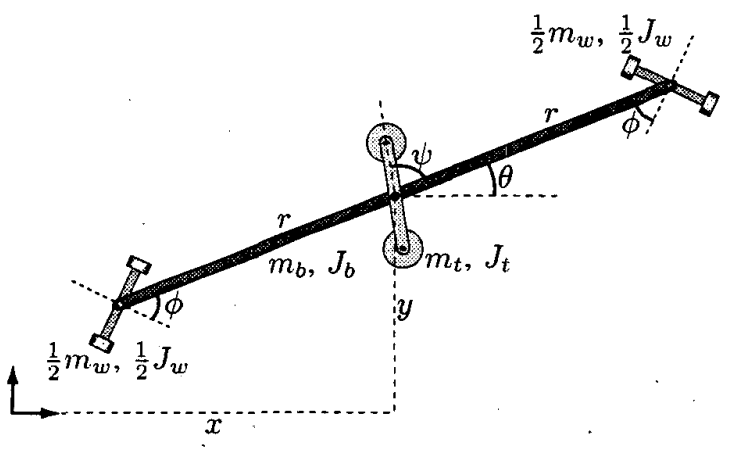

Fig. 1. Schematic (top) view of the snakeboard.

\section{THE SNAKEBOARD}

As an example of a nonholonomic mechanical system, we take the well-known and interesting system called the 'snakeboard'. Figure 1 shows a schematic view of it. This model has been analyzed many times before (see for example [6]), and represents a simplified version of the commercially available snakeboard [1], which is a locomotion device very similar to the skateboard, but with the ability to rotate the wheels at the front and the back of the board. Using only a combination of motion of these wheels and a rotation of the body (represented by the inertia $J_{t}$ ), it is possible to increase the forward momentum of the system (other maneuvers are also possible).

The model consists of three parts (body, torso and wheels), each with its own mass $m_{*}$ and inertia $J_{*}$. We define

$$
\begin{aligned}
m & :=m_{b}+m_{t}+m_{w} & & \text { total mass } \\
J & :=J_{b}+J_{t}+J_{w}+m_{w} r^{2} & & \text { total inertia around }(x, y)
\end{aligned}
$$

and make the simplifying assumptions that (1) the wheels rotate at equal but opposite angles (only one coordinate $\phi$ is used) and (2) the parameters are such that $J=J_{t}+m r^{2}$. Assumptions like these $e^{1}$ are standard in the analysis of the snakeboard and simplify the equations without affecting the essential geometry of the problem. Under these assumptions, the dynamic equations of the snakeboard can be written locally in symplectic PCHS form (6) and (7) with

$$
\begin{aligned}
q & =\left[\begin{array}{lllll}
x & y & \theta & \psi & \phi
\end{array}\right]^{T} \\
p & =\left[\begin{array}{lllll}
p_{x} & p_{y} & p_{\theta} & p_{\psi} & p_{\phi}
\end{array}\right]^{T} \\
B & =\left[\begin{array}{lllll}
0 & 0 & 0 & 1 & 0 \\
0 & 0 & 0 & 0 & 1
\end{array}\right]^{T} \\
H & =\frac{1}{2}\langle p, p\rangle_{g^{-1}} \\
g & =\left[\begin{array}{ccccc}
m & 0 & 0 & 0 & 0 \\
0 & m & 0 & 0 & 0 \\
0 & 0 & J & J_{t} & 0 \\
0 & 0 & J_{t} & J_{t} & 0 \\
0 & 0 & 0 & 0 & J_{w}
\end{array}\right]
\end{aligned}
$$

'The standard assumption is to take $J=m r^{2}$, but the equations simplify even further if we take $J=J_{t}+m r^{2}$.
The kinematic constraints present in this model are that the wheels are not allowed to slip, which implies that the velocities should belong to the distribution $\mathcal{D}$ given for example as

$$
\mathcal{D}(q):=\operatorname{span}\left\{r \cos (\theta) \partial_{x}+r \sin (\theta) \partial_{y}+\tan (\phi) \partial_{\theta}, \partial_{\psi}, \partial_{\phi}\right\}
$$

which corresponds to both relative internal degrees of freedom, as well as a rotation around the instantaneous center of rotation determined by the wheels. We assume that the relative angles of the wheels remain strictly between $\pm \frac{\pi}{2}$, in which case it can be checked that this distribution has constant dimension 3 and is not integrable.

\section{REDUCED PORT-HAMILTONIAN REPRESENTATIONS}

In this section, we transform the symplectic PCHS with nonholonomic constraints to a physically-interpretable reducedorder PCHS without constraints.

\section{A. Choice of Basis Vectors and New Coordinates}

To transform the dynamic equations (6) and (7) into a reduced-order model, we use the following coordinate transformation. First, we describe the constraints not in terms of velocities but in terms of momenta, i.e. we require that the momenta $p$ are in $\mathcal{C}_{q}^{\perp}$. Then, we choose a particular set of basis vectors $\left\{h^{a}(q)\right\}$ for $\mathcal{C}_{q}^{\perp}$, such that

- Every element $v \in \mathcal{C}_{q}^{\perp}$ can be written as a linear combination of $h^{a}(q)$ (i.e. it is a basis)

- The set of $\left\{h^{a}\right\}$ defines a diffeomorphism between $\mathbb{R}^{m}$ and $\mathcal{C}_{q}^{\perp}$ (where $m$ is the dimension of $\mathcal{C}_{q}^{\perp}$ ). In coordinates, the mapping $h_{i}^{a}$ relates $v \in \mathcal{C}_{\boldsymbol{q}}^{\perp}$ and $\alpha \in \mathbb{R}^{m}$ as

$$
\begin{aligned}
v_{i} & =h_{i}^{a} \alpha_{a} \\
\alpha_{a} & =\tilde{g}_{a b} h_{i}^{b} g^{i j} v_{j}
\end{aligned}
$$

where the second equation is actually the $g$-orthogonal projection on $\mathcal{C}_{q}^{\perp}$ for any element of $T_{q}^{*} Q$, and where

- The metric $\bar{g}$ on $\mathbb{R}^{m}$ induced by the metric $g$ and $h$, i.e.

$$
\bar{g}^{a b}(q)=h_{i}^{a}(q) g^{i j}(q) h_{j}^{b}(q)
$$

is diagonal and independent of $q$.

This choice of coordinates means that we will (locally) write the subbundle of $T^{*} Q$ satisfying the constraints as the product $Q \times \mathbb{R}^{m}$ with coordinates $(q, \alpha)$. Any element of this reduced space automatically satisfies the kinematic constraints. The energy $\bar{H}$ in these new coordinates can be written as

$$
\begin{aligned}
\bar{H}(q, \alpha) & :=H\left(q, h^{a} \alpha_{a}\right)=\frac{1}{2}\left\langle h^{a} \alpha_{a}, h^{b} \alpha_{b}\right\rangle_{g^{-1}}+V(q) \\
& =\frac{1}{2} \bar{g}^{a b} \alpha_{a} \alpha_{b}+V(q)=\frac{1}{2}\langle\alpha, \alpha\rangle_{\bar{g}^{-1}}+V(q)
\end{aligned}
$$

which is just the sum of potential energy and the kinetic energies of the components $\alpha$ in the directions defined by $h$. This decomposition essentially corresponds to a special choice of the matrix $S$ in the reduction procedure described in [10].

Since there are many possible choices for an orthogonal basis with constant norm, there are many choices for $h$. The right choice depends on the problem at hand. The choice of basis determines how the kinetic energy will be split into 
separate 'energy directions', and the problem will determine which directions are interesting to consider. For example, as shown in the next section, when looking at the snakeboard it is interesting to separate the direction of group motion to see how forward momentum can be increased.

A useful identity that follows from (9) is

$$
0=\bar{g}_{, k}^{a b}=h_{i, k}^{a} g^{i j} h_{j}^{b}+h_{i}^{a} g_{, k}^{i j} h_{j}^{b}+h_{i}^{a} g^{i j} h_{j, k}^{b}
$$

\section{B. Reduction to Minimal Coordinates}

Theorem 1: The mechanical system defined by (6) and (7) with constraints defined by the distribution $\mathcal{D} \subset T Q$ and $h$ as in the previous section can be written as

$$
\begin{aligned}
\frac{d}{d t}\left[\begin{array}{c}
q^{i} \\
\alpha_{a}
\end{array}\right] & =\bar{J}\left[\begin{array}{c}
\frac{\partial \bar{H}}{\partial q^{j}} \\
\frac{\partial \bar{H}}{\partial \alpha_{e}}
\end{array}\right]+\left[\begin{array}{c}
0 \\
\bar{g}_{a b} h_{i}^{b} g^{i j} B_{j}^{k}
\end{array}\right] u_{k} \\
y^{i} & =\left[\begin{array}{ll}
0 & B_{j}^{i} g^{j k} h_{k}^{a} \bar{g}_{a e}
\end{array}\right]\left[\begin{array}{c}
\frac{\partial \bar{H}}{\partial q^{j}} \\
\frac{\partial \bar{H}}{\partial \alpha_{e}}
\end{array}\right]
\end{aligned}
$$

where $\bar{H}(q, \alpha):=\frac{1}{2}\langle\alpha, \alpha\rangle_{\bar{g}^{-1}}+V(q)$ and

$$
\bar{J}:=\left[\begin{array}{cc}
0 & g^{i j} h_{j}^{a} \bar{g}_{a e} \\
-\bar{g}_{a e} h_{i}^{e} g^{i j} & \bar{g}_{a b} h_{i}^{b} g^{i j}\left(h_{k, j}^{c}-h_{j, k}^{c}\right) \alpha_{c} g^{k l} h_{l}^{d} \bar{g}_{d e}
\end{array}\right]
$$

Proof: We want to transform the dynamic equations in terms of $(q, p)$ coordinates to $(q, \alpha)$ coordinates. First note that from (8) and using (10), we can compute

$$
\begin{aligned}
& \frac{\partial \alpha_{a}}{\partial q^{i}}=-\bar{g}_{a b} h_{j}^{b} g^{j k} h_{k, i}^{c} \alpha_{c} \\
& \frac{\partial \alpha_{a}}{\partial p_{i}}=\bar{g}_{a b} h_{j}^{b} g^{i j}
\end{aligned}
$$

and hence

$$
\dot{\alpha}_{a}=-\bar{g}_{a b} h_{j}^{b} g^{j k} h_{k, i}^{c} \alpha_{c} \dot{q}^{i}+\bar{g}_{a b} h_{j}^{b} g^{j i} \dot{p}_{i}
$$

Along solutions of $(6,7)$ we have $\vec{H}(q, \alpha)=H(q, p)$. On this submanifold, we also have

$$
\begin{aligned}
& \frac{\partial H}{\partial q^{i}}=\frac{\partial \bar{H}}{\partial q^{i}}+\frac{\partial \bar{H}}{\partial \alpha_{a}} \frac{\partial \alpha_{a}}{\partial q^{i}} \\
& \frac{\partial H}{\partial p_{i}}=\frac{\partial \bar{H}}{\partial \alpha_{a}} \frac{\partial \alpha_{a}}{\partial p_{i}}
\end{aligned}
$$

Combining these results, we obtain

$$
\begin{aligned}
\dot{q}^{i} & =\frac{\partial H}{\partial p_{i}}=\frac{\partial \bar{H}}{\partial \alpha_{e}} \frac{\partial \alpha_{e}}{\partial p_{i}}=\frac{\partial \bar{H}}{\partial \alpha_{e}} \bar{g}_{a e} h_{j}^{a} g^{i j} \\
\dot{\alpha}_{a} & =-\bar{g}_{a b} h_{i}^{b} g^{i j} h_{j, k}^{c} \alpha_{c}\left(g^{k l} h_{l}^{d} \bar{g}_{d e} \frac{\partial \bar{H}}{\partial \alpha_{e}}\right) \\
& +\bar{g}_{a b} h_{i}^{b} g^{i j}\left(-\frac{\partial \bar{H}}{\partial q^{j}}+\frac{\partial \bar{H}}{\partial \alpha_{e}} \bar{g}_{d e} h_{l}^{d} g^{k l} h_{k, j}^{c} \alpha_{c}+B_{j}^{k} u_{k}\right) \\
y^{i} & =B_{j}^{i} \frac{\partial \hat{H}}{\partial \alpha_{a}} \frac{\partial \alpha_{a}}{\partial p_{j}}=B_{j}^{i} \frac{\partial \bar{H}}{\partial \alpha_{a}} \bar{g}_{a b} h_{k}^{b} g^{j k}
\end{aligned}
$$

where we used $h_{i}^{b} g^{i j} \mathcal{F}_{j}=0$ since $\mathcal{F} \in \mathcal{D}^{\perp}$. These equations can be expressed in matrix form as in the theorem.

\section{Reduced Snakeboard Model}

We now apply the results to the snakeboard. The first step to find the reduced equations is the choice of a useful constantnorm orthogonal basis for $\mathcal{C}_{q}^{\perp}$. Since we are interested in generating forward motion of the snakeboard, we choose to have one basis vector in the allowed group direction. The remaining two basis vectors are chosen to describe separately the momentum of the torso and the momentum of the wheels. Together, we choose the following mapping $h$ :

$$
h:\left[\begin{array}{c}
\alpha_{1} \\
\alpha_{2} \\
\alpha_{3}
\end{array}\right] \mapsto\left[\begin{array}{c}
\cos (\phi) \cos (\theta) \\
\cos (\phi) \sin (\theta) \\
r \sin (\phi) \\
0 \\
0
\end{array}\right] \alpha_{1}+\left[\begin{array}{l}
0 \\
0 \\
1 \\
1 \\
0
\end{array}\right] \alpha_{2}+\left[\begin{array}{l}
0 \\
0 \\
0 \\
0 \\
1
\end{array}\right] \alpha_{3}
$$

The induced metric on $\mathbb{R}^{3}$ can be represented by the matrix

$$
\bar{g}_{a b}=\left[\begin{array}{ccc}
m & 0 & 0 \\
0 & J_{t} & 0 \\
0 & 0 & J_{w}
\end{array}\right]
$$

so the coordinates $\alpha$ can be interpreted as momentum in the group direction (with inertia $m$ ), momentum of the torso (with inertia $J_{t}$ ) and momentum of the wheels (with inertia $J_{w}$ ). Since the metric $\bar{g}$ is diagonal and constant, this proves that indeed $h$ defines a constant orthogonal basis as required.

From this choice of $h$, we can compute the reduced dynamic equations for the snakeboard as

$$
\begin{aligned}
\frac{d}{d t} q & =\left[\begin{array}{ccc}
\cos (\phi) \cos (\theta) & 0 & 0 \\
\cos (\phi) \sin (\theta) & 0 & 0 \\
\frac{1}{r} \sin (\phi) & 0 & 0 \\
-\frac{1}{r} \sin (\phi) & 1 & 0 \\
0 & 0 & 1
\end{array}\right]\left[\begin{array}{c}
\frac{\alpha_{1}}{m} \\
\frac{\alpha_{2}}{J_{t}} \\
\frac{\alpha_{3}}{J_{w}}
\end{array}\right] \\
\frac{d}{d t} \alpha= & {\left[\begin{array}{cc}
-\frac{1}{r} \sin (\phi) & 0 \\
1 & 0 \\
0 & 1
\end{array}\right]\left[\begin{array}{l}
u_{1} \\
u_{2}
\end{array}\right] } \\
y & =\left[\begin{array}{ccc}
-\frac{1}{r} \sin (\phi) & 1 & 0 \\
0 & 0 & 1
\end{array}\right]\left[\begin{array}{l}
\frac{\alpha_{1}}{m} \\
\frac{\alpha_{2}}{J_{t}} \\
\frac{\alpha_{3}}{J_{w}}
\end{array}\right]
\end{aligned}
$$

which are remarkably simple. The energy part of the system (i.e. not including the first three equations for $\dot{q}$ ) can be conveniently represented ${ }^{2}$ by the bond graph of Figure 2 .

Though not commonly known, bond graphs (introduced by Paynter [11]) can be very useful to analyze energy aspects of physical systems. We give a rough guide how to read and use a bond graph like the ones in Figures 2 and 3; interested readers are referred to [12], [13] for a more accurate and complete introduction to bond graphs.

The half-arrows called bonds represent energy connections between subparts, carrying dual variables (called effort and flow, for mechanical systems force and velocity) where the dual product between the two represents the power flowing

${ }^{2}$ Even though $\phi$ is not the state of the wheel inertia, we use the arrow to denote that it can be computed from this state. The fact that $\bar{g}$ is constant allows us also to use regular II elements without parameter ports. 


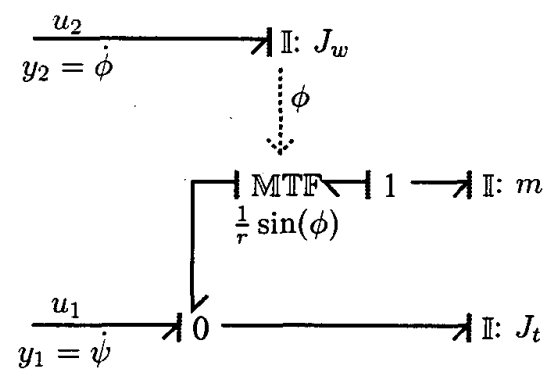

Fig. 2. Bond graph representation of the snakeboard.

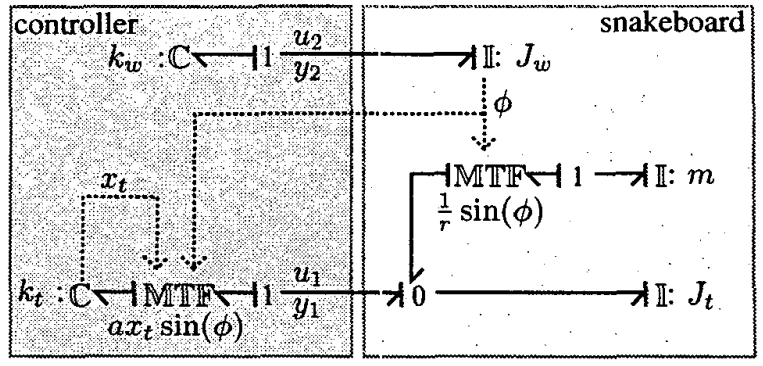

Fig. 3. Bond graph of the snakeboard and the controller. in the direction of the arrow. The stroke on either side of the arrow indicates the signal direction of the effort (force); the flow (velocity) is then in the opposite direction. The Is are inertial elements, which integrate the incoming effort (force) to get the internal state (momentum), and output the partial derivative of the energy function to the state (i.e. the velocity). Similarly, a $\mathbb{C}$-element represents an elastic element, integrating the incoming flow (velocity) to get the internal state (displacement), and output the partial derivative of the energy function to the state (i.e. elastic force). An MTFF-element (modulated transformer) establishes a powerconnection between two bonds, the coupling strength of which can be modulated by some external signal, in this case by $\sin (\phi)$. At all times, the power on the incoming bond is equal to the power on the outgoing bond. Finally, 0 - and 1-junctions represent generalized Kirchhoff laws, i.e. all connecting bonds on a 0 -junction have equal effort, all connecting bonds on a 1 -junction have equal flow, and the (signed) sum of the power on the bonds equals zero.

The bond graph in Figure 2 shows that the wheels (II with inertia $J_{w}$ ) do not have an energy coupling to the rest of the snakeboard. They only influence the system indirectly through modulation of the MTF. The input port $\left(u_{1}, \dot{\psi}\right)$ can exchange energy with the torso (II with inertia $J_{t}$ ) as well as with the group motion (II with inertia $m$ ), but only if $\sin (\phi) \neq 0$. All of this is clear from intuitive physical reasoning about the snakeboard (e.g. if the wheels are straight $(\phi=0)$, exerting a torque $u_{1}$ only makes the torso rotate, not the rest of the snakeboard); the reduced PCHS and the corresponding bond graph prove this reasoning and quantify it.

\section{ENERGY-BASED CONTROL OF THE SNAKEBOARD}

In this section, we describe how the PCHS and its corresponding bond graph can be used to construct a physically motivated controller for the snakeboard. We present just one example of such a controller; of course many extensions, modifications and optimizations are possible. Note that contrary to common work, we have not used any linearizing or other feedback; we are still working with the same bare nonlinear system, only expressed in different coordinates.

We only look at the control problem of generating group motion (forward motion with possibly changing direction) of the snakeboard, i.e. we are not interested in general gaits (like rotation or parallel parking). With the model described in the previous section, this goal can be reformulated as: how can we, starting from rest, increase the energy in the $\alpha_{1}$ component, i.e. energy in the $\mathbb{I}$ element with inertia $m$.

Since there is only an energy coupling to the $\alpha_{2}$ component for nonzero $\phi$, we cannot take $\phi=0$ even though this is desired for straight motion. Instead, we make $\phi$ oscillate periodically (around a point depending on the desired direction) by connecting a torsional spring to the port $\left(u_{2}, y_{2}\right)$, i.e. between the wheels and the frame.

We propose the following controller structure

$$
\begin{aligned}
\frac{d}{d t}\left[\begin{array}{l}
x_{t} \\
x_{w}
\end{array}\right] & =\left[\begin{array}{ll}
0 & 0 \\
0 & 0
\end{array}\right]\left[\begin{array}{l}
\frac{\partial H_{c}}{\partial x_{t}} \\
\frac{\partial H_{c}}{\partial x_{u}}
\end{array}\right]+\left[\begin{array}{cc}
a x_{t} \sin (\phi) & 0 \\
0 & 1
\end{array}\right]\left[\begin{array}{l}
y_{1} \\
y_{2}
\end{array}\right] \\
{\left[\begin{array}{l}
-u_{1} \\
-u_{2}
\end{array}\right] } & =\left[\begin{array}{cc}
a x_{t} \sin (\phi) & 0 \\
0 & 1
\end{array}\right]\left[\begin{array}{l}
\frac{\partial H_{c}}{\partial x_{t}} \\
\frac{\partial H_{c}}{\partial x_{w}}
\end{array}\right]
\end{aligned}
$$

with $a>0$ a parameter, and $H_{c}\left(x_{t}, x_{w}\right)=\frac{1}{2} k_{t} x_{t}^{2}+\frac{1}{2} k_{w} x_{w}^{2}$, i.e. two independent springs, one (with state $x_{w}$ ) connected directly, and one (with state $x_{t}$ ) connected through a modulated transformer. The corresponding bond graph of this controller as connected to the snakeboard is shown in Figure 3. The dynamic equations of the closed-loop system become

$$
\begin{aligned}
& \frac{d}{d t}\left[\begin{array}{l}
\alpha_{3} \\
x_{w}
\end{array}\right]=\left[\begin{array}{cc}
0 & -1 \\
1 & 0
\end{array}\right]\left[\begin{array}{c}
\frac{\alpha_{3}}{J_{w}} \\
k_{w} x_{w}
\end{array}\right] \\
& \frac{d}{d t}\left[\begin{array}{l}
\alpha_{1} \\
\alpha_{2} \\
x_{t}
\end{array}\right]=\left[\begin{array}{ccc}
0 & 0 & \frac{1}{r} a x_{t} \sin ^{2}(\phi) \\
0 & 0 & -a x_{t} \sin (\phi) \\
-\frac{1}{r} a x_{t} \sin ^{2}(\phi) & a x_{t} \sin (\phi) & 0
\end{array}\right]\left[\begin{array}{c}
\frac{\alpha_{1}}{m} \\
\frac{\alpha_{2}}{J_{t}} \\
k_{t} x_{t}
\end{array}\right]
\end{aligned}
$$

which shows that the inertia of the wheels connected to the spring $k_{w}$ forms a harmonic oscillator, indeed making $\phi$ change periodically. Furthermore, we can see that $\dot{\alpha}_{1} \geq 0$, i.e. as long as $x_{t} \neq 0$ and whenever $\phi \neq 0$, the energy in the group direction $\alpha_{1}$ increases. However, also some energy (at least temporarily) flows to the $\alpha_{2}$ component (torso motion).

So if we start the controller from rest with some initial energy in the controller states $x_{t}$ and $x_{w}$, then the energy in $x_{w}$ will oscillate back and forth periodically between $x_{w}$ and $\alpha_{3}$, and all other energy will eventually leave $x_{t}$ and be stored in the inertial elements $\alpha_{1}$ and $\alpha_{2}$ (energy in the group and energy in the torso), where the exact splitting between these two states depends on the initial conditions. We use 




Fig. 4. Evolution of the state trajectory on an elljpsoid of constant energy.

a simulation (Figure 4) to show how the states $\left(\alpha_{1}, \alpha_{2}, x_{t}\right)$ evolve over time for certain initial conditions. Starting from an initial state with all energy in the controller (and velocities zero), we see how this energy is transferred to the group momentum, increasing $\alpha_{1}$ to a maximum. The total energy is constant at all times, which can be seen in the simulation plot; the state evolves on an ellipsoid of constant energy, given by the equation

$$
\frac{1}{2}\left(\frac{\alpha_{1}^{2}(\tau)}{m}+\frac{\alpha_{2}^{2}(\tau)}{J_{t}}+k_{t} x_{t}^{2}(\tau)\right)=\frac{1}{2} k_{t} x_{t}^{2}(0) \quad \forall \tau \geq 0
$$

As seen in the simulation, for these initial conditions all energy (i.e. excluding the energy flowing between $\alpha_{3}$ and $x_{w}$, the wheels) is transferred eventually to the group direction $\alpha_{1}$.

\section{CONCLUSIONS AND FUTURE WORK}

We have shown how a mechanical systems with nonholonomic kinematic constraints can be transformed into a reduced-order model without constraints described as a PortControlled Hamiltonian System. Depending on a choice of basis vectors for the codistribution of allowed momenta, the kinetic energy can be written as the sum of the individual energies associated with the directions of these momenta.

By making the right choice of basis, the equations show how energy flows between the different directions, and how for example control forces can influence these energy flows. In this way, the presented method can be a useful tool for analysis purposes.

The method was demonstrated on the snakeboard, and by choosing some basis vectors related to internal (shape) energy and some to external (group) energy, we derived a very simple representation of the dynamics of the snakeboard, explicitly showing the energy coupling between inputs and momenta. No linearizing feedback or other changes were applied. The model was then used to intuitively design an energy-based controller that generated forward motion.

The example of the snakeboard showed how the energybased reduced model of Section IV can be used as a starting point to design controllers that are physically motivated and have a clear energy concept. In practical situations where damping is present, these controllers will never be sufficient by themselves, but they can provide a starting point for the design of efficient controllers (e.g. in locomotion devices), which on one hand contain passive elements like springs to generate the basic behavior of the oscillations (like a torsional spring on the wheels of the snakeboard), and on the other hand have motors that supply the energy lost to dissipation and correct for modeling errors and disturbances.

In future work (see [14]), we would like to analyze the geometric structure of the reduced model in more detail, especially the meaning of the bottom-right skew symmetric term in $\bar{J}$ in (11). We also want to find the precise relationship between the momentum equations for the momentum in the group direction and the well-known (nonholonomic) momentum maps often used in literature.

Finally, we want to apply the results to the analysis and control of walking robots, which means the method will have to be extended for systems with discrete events and statejumps, which are commonly used to model the impact of the feet with the ground.

\section{ACKNOWLEDGMENTS}

This work has been done in the context of the European sponsored project GeoPleX with reference code IST-2001-34166. Further information is available at http://www.geoplex.cc.

\section{REFERENCES}

[1] Snakeboard U.S.A., http://www.snakeboard.com.

[2] A. Lewis, J. Ostrowski, R. Murray, and J. Burdick, "Nonholonomic Mechanics and Locomotion: the Snakeboard Example," in Proceedings of the IEEE Conference on Robotics and Automation, 1994.

[3] F. Bullo and M. Zefran, "On Mechanical Control Systems with Nonholonomic Constraints and Symmetries," Systems and Control Letters, vol. 45, no. 1, pp. 133-143, 2001.

[4] P. Vela, "Averaging and Control of Nonlinear Systems," Ph.D. dissertation, California Institute of Technology, 2003.

[5] P. Vela, K. Morgansen, and J. Burdick, "Second-order Averaging Methods and Oscillatory Feedback Control of Underactuated Mechanical Systems," in Proceedings of the IEEE American Control Conference, 2002, pp. 4672-4677.

[6] A. Bloch, Nonholonomic Mechanics and Control, ser. Interdisciplinary Applied Mathematics (24). Springer-Verlag, 2003.

[7] G. Blankenstein, "Symmetries and Locomotion of a 2D Mechanical Network: the Snakeboard," in Lecture Notes for the Euron/GeoPleX Summer School. Bertinoro, Italy, July 2003.

[8] A. v. d. Schaft, $L_{2}$-Gain and Passivity Techniques in Nonlinear Control, ser. Communications and Control Engineering. Springer-Verlag, 2000.

[9] A. Isidori, Nonlinear Control Systems. Springer-Verlag, 1995.

[10] A. v. d. Schaft and B. Maschke, "On the Hamiltonian Formulation of Nonholonomic Mechanical Systems," Reports on Mathematical Physics, vol. 34, pp. 225-233, 1994.

[11] H. Paynter, Analysis and Design of Engineering Systems. M.I.T. Press, 1961.

[12] D. Karnopp, D. Margolis, and R. Rosenberg, System Dynamics, a Unified Approach. John Wiley and Sons, 1990.

[13] S. Stramigioli, Modeling and IPC Control of Interactive Mechanical Systems - A Coordinate-free Approach. Springer-Verlag, 2001.

[14] V. Duindarn, G. Blankenstein, and S. Stramigioli, "Port-Based Modeling and Analysis of Snakeboard Locomotion," 2004, submitted to the International Symposium on Mathematical Theory of Networks and Systems. 